\title{
A Labview Based Leakage Current Monitoring System For HV Insulators
}

\author{
N. Mavrikakis*,', I. Androulidakis ${ }^{2}$, D. Pylarinos ${ }^{1}$ and K. Siderakis ${ }^{1}$ \\ ${ }^{I}$ Department of Electrical Engineering, Technological Educational Institute of Crete, Greece. \\ ${ }^{2}$ University of Ioannina, Greece.
}

Received 28 October 2015; Accepted 1 December 2015

\begin{abstract}
In this paper, a Labview based leakage current monitoring system for High Voltage insulators is described. The system uses a general purpose DAQ system with the addition of different current sensors. The DAQ system consists of a chassis and hot-swappable modules. Through the proper design of current sensors, low cost modules operating with a suitable input range can be employed. Fully customizable software can be developed using Labview, allowing on-demand changes and incorporation of upgrades. Such a system provides a low cost alternative to specially designed equipment with the added advantage of maximum flexibility. Further, it can be modified to satisfy the specifications (technical and economical) set under different scenarios. In fact, the system described in this paper has already been installed in the HV Lab of the TEI of Crete whereas a variation of it is currently in use in TALOS High Voltage Test Station.
\end{abstract}

Keywords: Labview, High Voltage, insulators, leakage current, monitoring, lab, field, sensors, DAQ

\section{Introduction}

Leakage current monitoring is a widely employed technique for monitoring and investigating the behaviour and performance of high voltage insulators [1-5]. Surface discharge activity, which is illustrated in the leakage current waveform, is linked to the pollution accumulation, the aging of polymer insulators and the advance towards flashover [14]. However, leakage current recording and the interpretation of data can be a demanding task as a fully representative value of the shape of the waveform is yet to be proposed. Several extracted values are commonly used [5] and advanced pattern recognition techniques have also been proposed [6-7]. Further, the size of the gathered data [8] and noise is always an issue [9]. Therefore, a leakage current monitoring system should be as flexible as possible in order to cope with different testing scenarios and to be able to incorporate future processing techniques. This paper describes the Labview based approach to leakage current monitoring, that the High Voltage Lab of the Electrical Engineering Department of the Technological Educational Institute of Crete in Iraklion, Crete is following. A similar approach is also followed in HEDNO's TALOS High Voltage Test Station in Iraklion, Crete [10].

\section{The Labview Approach}

A few devices specially designed for leakage current are commercially available (e.g. [11-13]) but the nature of the application has lead several labs and utilities to start developing their own prototypes (e.g. [14]). A tradeoff between cost and capabilities has to be made in any case.
This paper describes a novel approach to the problem: the use of Labview based general purpose DAQs.

The basic form of a leakage current monitoring system consists of current sensors, a data acquisition kit and a storage device. The monitoring system discussed in this paper, is largely based on commercially available general purpose equipment, in order to provide a low-cost solution offering increased flexibility and adaptability. The scheme followed employs the use of a rugged and reconfigurable chassis equipped with a microprocessor and userprogrammable FPGA and multiple hot-swappable I/O modules. Custom made VIs are developed for processing, recording and depicting the data. Modified commercially available sensors are also employed. The first version of the system is presented along with the first recording results.

\section{Current Monitoring System}

The setup of the designed system allows the flexible combination of different components, based on a Labview compatible system. Different types of current sensors or data acquisition cards can be used in that system whereas the software performing the signal processing part is fully customizable, as well. The fundamental principle is that the leakage current is converted to a voltage signal by the sensor. This voltage signal is then sent to the Data Acquisition device (DAQ). In case of a PC-based system, the data are sent to a PC which storages them and/or sends them through a network to other stations or uploads them in a cloud database (Fig. 1). If the DAQ is equipped with an onboard memory then a stand-alone operation can be achieved [15].
* E-mail address: mavrikakisnikos@gmail.com ISSN: 1791-2377 @ 2015 Kavala Institute of Technology. All rights reserved. 


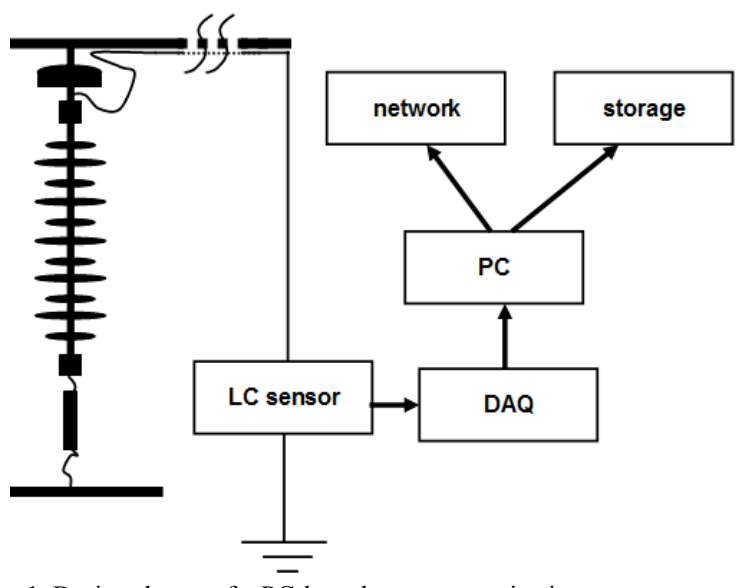

Fig. 1. Basic scheme of a $\overline{P C}$-based current monitoring system

\section{Current sensors}

\subsection{Hall sensors with a $\pm 15 \mathrm{~V}$ output}

The sensors described here were part of a no-longer commercially available leakage current device [16] that was purchase in the past by HEDNO. Through the cooperation with HEDNO [17], some of these sensors were made available for laboratory purposes. These sensors were based on a Hall current sensor and they were designed to be placed outdoors in a large distance from the DAQ. Therefore, they were properly shielded and incorporated signal amplification suitable for large distances. However, their main disadvantage was their $\pm 15 \mathrm{~V}$ output range which is larger than the usual input range used by general purpose DAQs $( \pm 10 \mathrm{~V})$. This meant that special DAQs with a larger input range had to be ordered in order to use these sensors (leading to a higher cost). A picture of the internals of such a sensor is shown in Fig. 2. Considering that sensors are now housed even in TALOS [15], that the DAQ originally equipped with these sensors is out-of-duty and that a significant cost reduction will be achieved if a lower output range is provided, a decision was made to develop new sensors. Several approaches are currently being tested and a finished and operational prototype is described in the following paragraph.

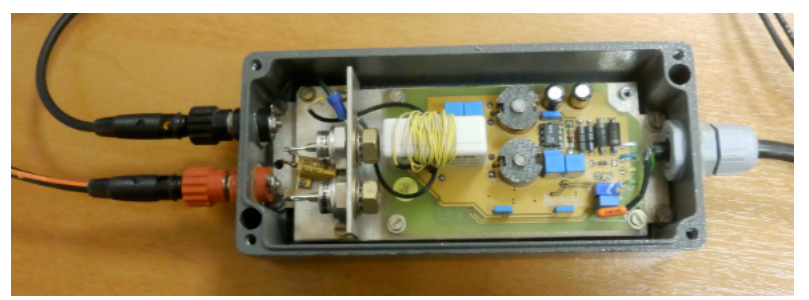

Fig. 2. The Hall sensor with a $\pm 15 \mathrm{~V}$ output range

\subsection{Current sensor with a $\pm 10 \mathrm{~V}$ output}

A photograph of this sensor is shown in Fig. 3). It is built around a current sensor driving an operational amplifier. The following specifications were set for the sensor: $1 \mathrm{~mA}$ current resolution, $1 \mathrm{~mA}$ to $1.5 \mathrm{~A}$ current range and a $1 \mathrm{~mA}$ to $10 \mathrm{mV}$ current to voltage conversion (10x gain). The current sensor was based on IM120710018 "electronic brick" from ITEAD studio [18]. An electronic brick is an electronic module which can be assembled like Lego bricks simply by plugging in and pulling out. The given "electronic brick" of the sensor itself is based on TA12-100 current transformer, which can transform large current $\mathrm{AC}$ signals into smaller amplitude signals outputting the signal read via the analog I / O port. The transformer has a 1000:1 windings ratio, and can accept a 0 to $5 \mathrm{~A}$ input, yielding a respective output of 0 to $5 \mathrm{~mA}$. The primary current to be measured is fed via a single wire passing through the aperture in the center of the transformer as seen in Fig. 3. The protection diodes are on the right. The sensing current wire is seen on the center of the image, passing via the aperture of the transformer
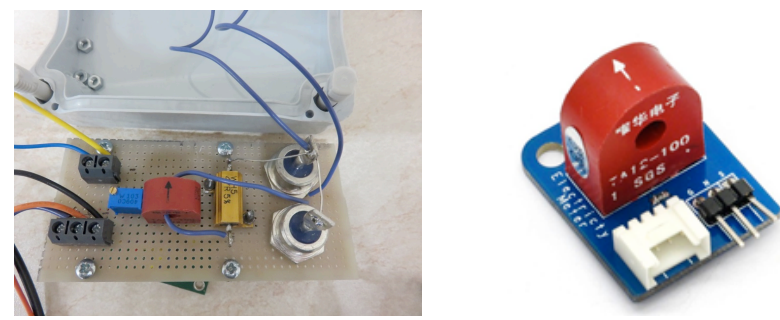

Fig. 3. The upper side of the hardware and the current sensor.

A simple circuit is used to measure the inductive current via the respective voltage drop in a shunt resistor, using an operational amplifier in a negative feedback (non-inverting) connection. To that extend, the Texas Instruments DEMOPA-SO-1A demonstration fixture, which is a generic, unpopulated printed circuit board (PCB) for single operational amplifiers in SO-8 packages, was populated with the TL071CD Texas Instruments JFET-Input, low noise operational amplifier [19]. The exact schematic diagram of DEM-OPA-SO-1A can be found in [20]. R1 was $1 \mathrm{kOhm}$, $\mathrm{R} 2$ and R7 were short-circuited (zero ohm resistors) and R3, R4, R8-R13 were left unpopulated. The resistor network of R5-R6 was formed using a $10 \mathrm{kOhm} 25$ turn trimmer, providing adjustable gain. Closing, an overvoltage protection block was placed in front of the circuit, using two 70 Amp, $1600 \mathrm{~V}$ Vishay Diodes, as seen on the right side of Fig. 3.

\section{Data Acquisition Kit}

The data acquisition kit consists of a NI Compact cDAQ9184 [21] chassis equipped with a NI 9221 [22] and a NI 9205 [23] module (Fig. 4). As a general scheme, the module conditions and digitizes the input signals and the chassis controls the timing and data for the acquisition system.

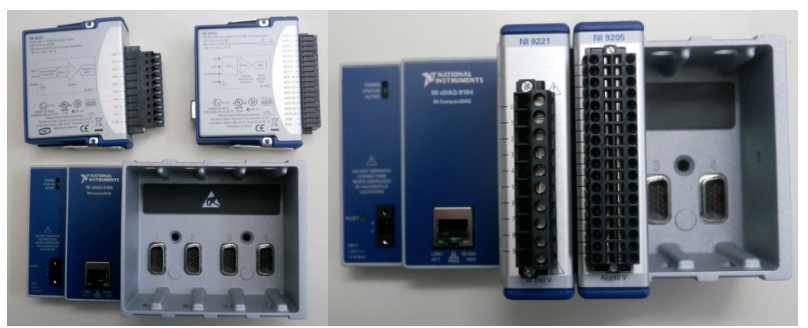

Fig. 4. NI Compact DAQ 9184 chassis equipped with a NI 9221 and a NI 9205 module

The NI 9221 module has 8 single ended inputs with an input range of $\pm 60 \mathrm{~V}$ input so it can be connected to the sensors with a $\pm 15 \mathrm{~V}$ output. The single ended feature portrays minimal problems for lab measurements while providing extra channels for a lower price (however, in case of field measurements a differential channel module like NI 9229 is preferred [15]). The NI 9205 module has 32 single ended channels (or 16 differential channels) with an input 
range of $\pm 10 \mathrm{~V}$ and therefore can only be used with sensors with such an output range (as are the ones described in the previous paragraph).

The advantage of using the Ethernet chassis over common USB data acquisition cards is that it allows taking long distance measurements and cooperates with remote or distributed measurement applications via the local network. Both of them are required when the application aims to the live and on-line monitoring of high voltage insulators. In addition the 4 slot inputs of the Ethernet chassis in combination with its multiple timing engines provide the opportunity of system scalability, which means that different types of sensors can be connected (e.g. weather sensors) and each channel can be sampled with a different rate.

An experimental set-up employed to test the system is shown in Fig. 5. A stand-alone capability can be achieved if additional Labview tools are purchased [15]. However, in such a case a different approach in the developed software should be followed due to the differences in the internal operation of the chassis in stand-alone mode (e.g. the DAQ block used in the second case does not come with an incorporated buffer).

\section{Software}

Custom made VIs were developed in order to acquire the measurements, process the signal, extract and record certain values and also the waveform (if required). Figure 6 shows an example of a custom VI employed for live leakage current monitoring during an inclined plane test. The IEC 60587 [24] was followed for the test set up. Apart from leakage current, the applied high voltage waveform and the aging time are recorded. This VI also gives the capability of visual observation by using a web camera through the Labview vision library. In addition the main frequency components and the root mean square values (RMS) of leakage currents are calculated and monitored. Live overcurrent warning/alarm providing the opportunity of fully controlled test parameters is also incorporated. All data are acquired, calculated, processed and finally stored in a network disk connected with a modem to the local network. Finally, the software can be compiled to an executable program by using additional Labview tools, thus providing the advantages of a user friendly stand-alone application without requiring programming expertise.

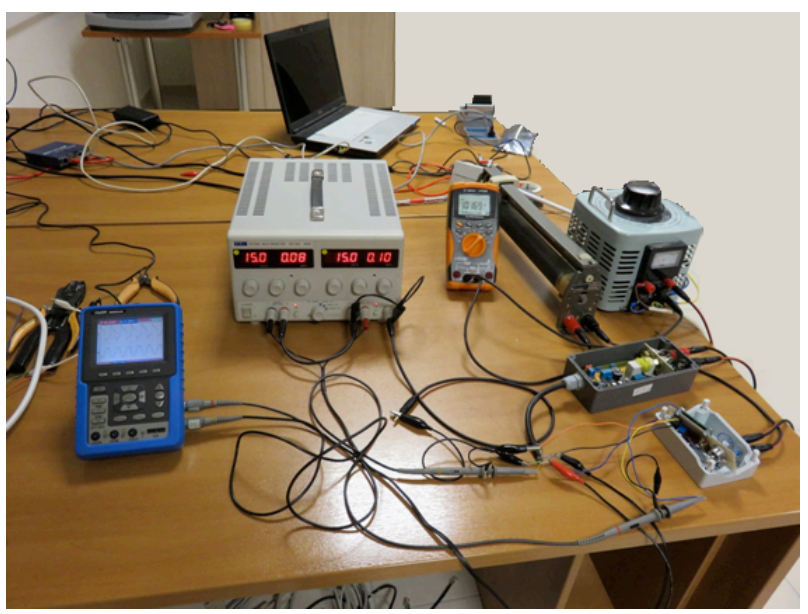

Fig. 5. The experimental set-up employed to test the system

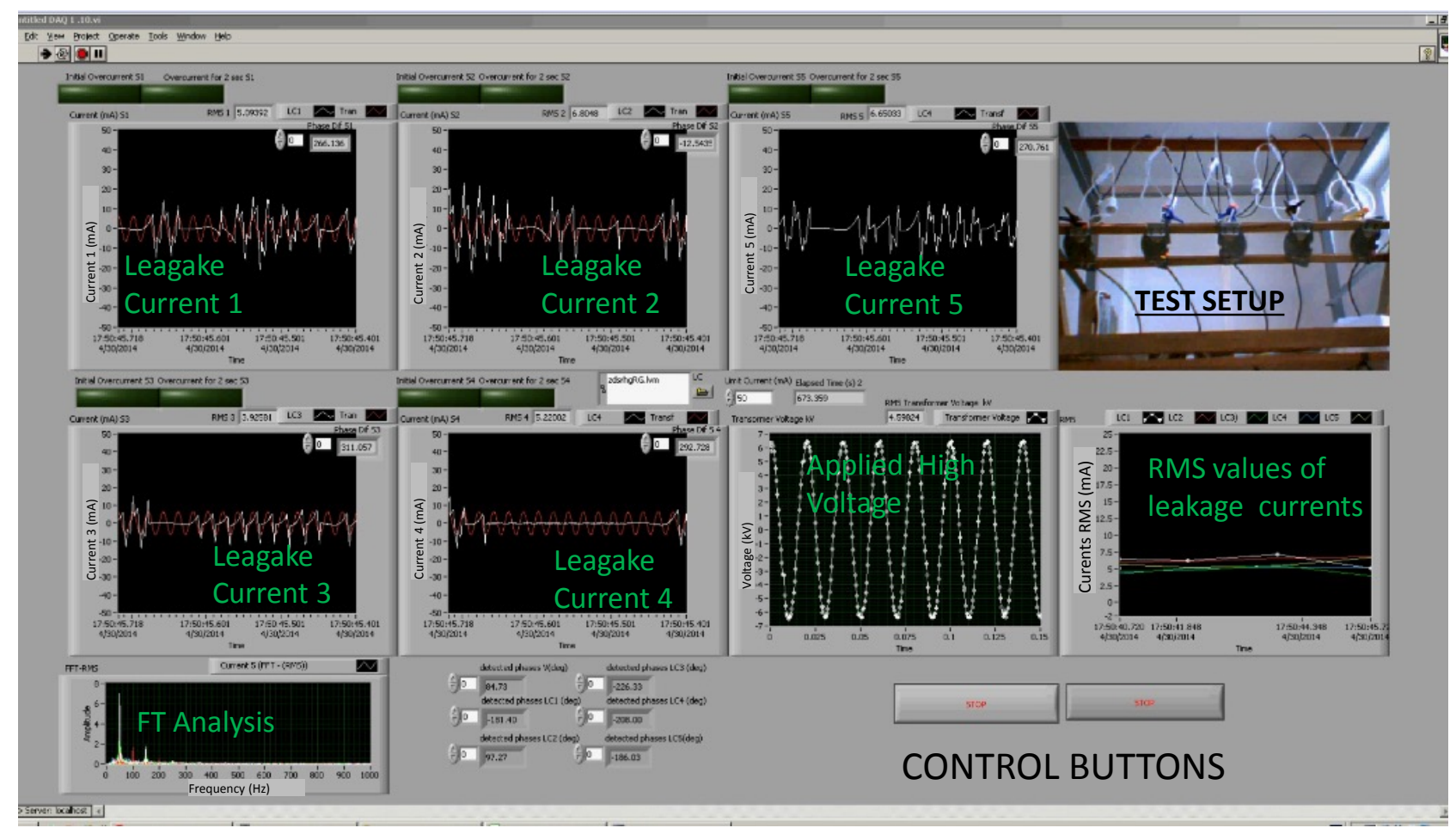

Fig. 6. A screenshot of a custom VI developed for LC monitoring

\section{Future work}

As research progresses, different sensors will be purchased, developed, tested and evaluated. Two types of current sensors [25-26] that are currently under evaluation are shown in Fig. 7. The goal is to identify an optimum solution regarding the cost, availability and performance. Further, different approaches for the system as a whole (or for certain 
parts) will be employed in order to test and evaluate their performance under different scenarios and specifications (e.g. a system to be installed in a test station, a system to be installed on Transmission towers etc)

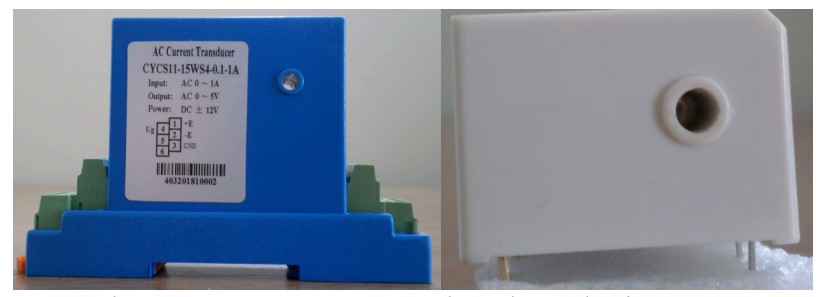

Fig. 7. The two current sensors currently under evaluation

\section{Conclusion}

Leakage current monitoring is a well established technique to monitor the behaviour and performance of high voltage insulators. Leakage current monitoring may require different approaches under different scenarios. This can be appointed due to practical reasons (noise, data size etc) but also due to the level of the desired analysis. Further, the recording of additional signals (e.g. weather data) with different sampling rates may be required. This paper describes a Labview based approach for leakage current monitoring that can be employed in order to develop a low-cost, fully customizable monitoring system with maximum flexibility which, given the nature of the task, is a major advantage over commercially available monitoring devices. Two different variations of such a system are currently in use (one in the lab and the other in a test station). Results show that the described general scheme is a rather promising one. Ongoing research is currently focused in optimizing the system, evaluating different approaches on certain aspects (e.g. Labview software development, current sensors, DAQs, stand-alone capability etc) and considering the different specifications and limitations set under different scenarios. The final goal is to gain an insight on the advantages and disadvantages of several different pieces (newly developed or commercially available) of general purpose hardware used to develop leakage current monitoring systems equipped with fully customized Labview based software capable of fulfilling the specifications (technical and economical) of different scenarios.

\section{Acknowledgements}

The authors would like to thank Dr. Georgios Sidiropoulos for his valuable contribution during the sensor prototype design and development.

This work was supported by the Polydiagno research project (project code 11SYN-7-1503) which is implemented through the Operational Program "Competitiveness and Enterpreneurship". Action "Cooperation 2011" and is cofinanced by the European Union and Greek national funds (National Strategic Reference Framework 2007-2013).

\section{References}

1. CIGRE WG 33-04, TF 01, A review of current knowledge: polluted insulators, CIGRE, 1998

2. D. Pylarinos, K. Siderakis, E. Pyrgioti "Measuring and analyzing leakage current for outdoor insulators and specimens", Reviews on Advanced Materials Science, Vol. 29, No. 1, pp. 31-53, 2011

3. IEC/TS 60815, Selection and dimensioning of high-voltage insulators intended for use in polluted conditions, International Electrotechnical Commission, 2008

4. CIGRE WG C4.303, Outdoor insulation in polluted conditions: Guidelines for selection and dimensioning, CIGRE, 2008

5. CIGRE TF 33.04.07, Natural and artificial ageing and pollution testing of polymeric insulators, CIGRE Technical Brochure, No. 142, 1999

6. D. Pylarinos, K. Theofilatos, K. Siderakis, E. Thalassinakis, I. Vitellas, A. T. Alexandridis, E. Pyrgioti, "Investigation and classification of field leakage current waveforms", IEEE Transactions on Dielectrics and Electrical Insulation, Vol. 19, No. 6, pp. 21112118,2012

7. K. Theofilatos, D. Pylarinos, S. Likothanassis, D. Melidis, K. Siderakis, E. Thalassinakis, S. Mavroudi, "A Hybrid Support Vector Fuzzy Inference System for the Classification of Leakage Current Waveforms Portraying Discharges", Electric Power Components \& Systems, Vol. 42, No. 2, pp. 180-189, 2014

8. D. Pylarinos, K. Siderakis, E. Thalassinakis, E. Pyrgioti, I. Vitellas, S. L. David, "Online applicable techniques to evaluate field leakage current waveforms", Electric Power Systems Research, Vol. 84, No. 1, pp. 65-71, 2012

9. D. Pylarinos, K. Siderakis, E. Pyrgioti, E. Thalassinakis, I. Vitellas, "Impact of noise related waveforms on long term field leakage current measurements", IEEE Transactions on Dielectrics and Electrical Insulation, Vol. 18, No. 1, pp. 122-129, 2011

10. TALOS High Voltage Test Station, www.talos-ts.com

11. WESTCO, Insulator Pollution Monitor, http://westcophil.com/wp/wp-content/uploads/IPMBrochure.pdf
12. MagShield, Leakage Current Monitoring System, http://www.magshield.com.au/lcmsfly.htm

13. PSP inc, Insulator Leakage Current Monitor,
$\mathrm{http}: / /$ www.psptechnologies.com/Assets/nonhtml/articulo_1_ingles.p http://www.psptechnologies.com/Assets/nonhtml/articulo_1_ingles.p

14. G. Zhicheng, Monitoring Leakage Current to Evaluate Insulator Pollution, INMR, 2014, http://www.inmr.com/monitoring-leakagecurrent-evaluate-insulator-pollution/

15. D. Pylarinos, N. Mavrikakis, K. Siderakis, E. Thalassinakis, "R\&D in TALOS High Voltage Test Station - A Distributed Approach on Insulator Monitoring Systems", 3rd Panhellenic Conference on Electronics and Telecommunications, Ioannina, Greece, May 8-9, 2015

16. CTLab, OLCA Product Description, http://www.ctlab.com/ downloads/all\%20olca\%20new.pdf

17. POLYDIAGNO, http://sinergasia.iesl.forth.gr/

18. ITEAD STUDIO, Electronic Brick - Electricity Meter (analog), Model: IM120710018 http://imall.iteadstudio.com/ im120710018.html

19. Texas Instruments , TL07x Low-Noise JFET-Input Operational Amplifiers, http://www.ti.com/lit/ds/symlink/tl071a.pdf

20. http://www.ti.com/lit/ug/sbou009a/sbou009a.pdf

21. Nantional Instruments, cDAQ-9184, http://sine.ni.com/nips/cds/ view/p/lang/en/nid/210669

22. National Instruments, NI 9221, http://sine.ni.com/nips/cds/ view/p/lang/en/nid/208799

23. National Instruments, NI 9205, http://sine.ni.com/nips/cds/ view/p/lang/en/nid/208800

24. IEC 60587, Electrical insulating materials used under severe ambient conditions - Test methods for evaluating resistance to tracking and erosion, 2007

25. ChenYang, AC Current Sensor CYCS11-xnWS4, http://www.cysensors.com/CYCS11-xnWS4.pdf

26. ChenYang, High Accurate AC Current Sensor CYCS411D47, http://www.cy-sensors.com/CYCS411D47.pdf 